\title{
White dwarf cosmochronology: Techniques and uncertainties
}

\author{
Maurizio Salaris \\ Astrophysics Research Institute, Liverpool John Moores University, \\ 12 Quays House, Birkenhead, CH41, 1LD, UK \\ email: ms@astro.livjm.ac.uk
}

\begin{abstract}
White dwarfs represent the endpoint of the evolution of the large majority of stars formed in the Galaxy. In the last two decades observations and theory have improved to a level that makes possible to employ white dwarfs for determining ages of the stellar populations in the solar neighborhood, and in the nearest star clusters. This review is centered on the theory behind the methods for white dwarf age-dating, and the related uncertainties, with particular attention paid to the problem of the CO stratification, envelope thickness and chemical composition, and the white dwarf initial-final-mass relationship.
\end{abstract}

Keywords. convection, dense matter, stars: atmospheres, stars: interiors, white dwarfs

\section{Introduction}

White dwarfs (WDs) are the last evolutionary phase of stars with initial masses smaller than about 10-11 $M_{\odot}$. The large majority of WDs, i.e. with progenitor masses below $\sim 6-7 M_{\odot}$, have masses between $\sim 0.55$ and $\sim 1.0 M_{\odot}$ and are made of an electron degenerate core of carbon and oxygen. Higher mass WDs have an oxygen and neon core. Some WDs originated by low-mass progenitors stripped of their envelope during the Red Giant Branch phase (e.g. in a binary system) have masses below $0.5 M_{\odot}$ and are made of helium. In all cases, the electron degenerate core is surrounded by a layer of pure He with mass of the order of $M_{H e} \sim 10^{-2} M_{W D}$ or less. This He-layer can be, in turn , surrounded by a H-layer with mass of the order of $M_{H} \sim 10^{-4} M_{W D}$ or less. Due to very efficient atomic diffusion, all metals in the envelope during the progenitor's evolution have settled at the bottom of the He-layer. WDs are spectroscopically labelled as DA if they have essentially a pure-H outer envelope, or non-DA in case of no hydrogen in the outer layers. Among the non-DA WDs the DB subclass corresponds to a pure He-envelope. The number ratio non-DA/DA appears to change with temperature for WDs in the solar neighborhood. For the range of luminosities of interest here, Tremblay \& Bergeron (2008) find empirically an increase of the non-DA/DA ratio when $T_{\text {eff }}$ decreases below $\sim 10000 \mathrm{~K}$, which is ascribed to mixing of a thin $\mathrm{H}$-envelope with the more massive underlying He-layers. Other types of non-DA objects, which typically appear at low $T_{\text {eff }}$, arise probably from convective mixing of the He-layer with underlying metals, and/or accretion of metals from the interstellar medium. This review will be focused on WDs with a carbon oxygen $(\mathrm{CO})$ core and will consider DB models as templates for the evolution of non-DA WDs.

The basic structure of a WD is simple. Almost all WD mass is in the CO core, that is nearly isothermal, because of the high electron conductivity. The WD evolution is a cooling process, whereby the core acts as energy reservoir (the energy available to be radiated away is the internal energy of the $\mathrm{CO}$ ions), the outer non-degenerate layers control the rate of energy outflow, and the luminosity and core temperature decrease 

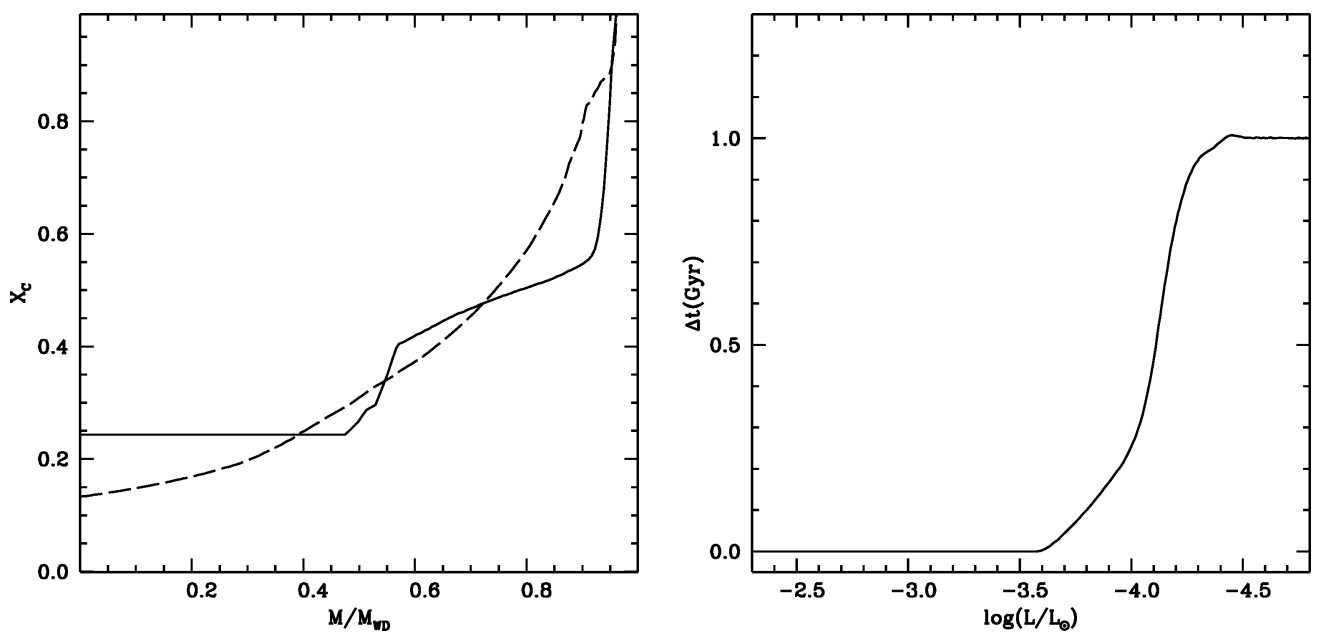

Figure 1. Left : Initial carbon abundance profile in the core of a $0.61 M_{\odot}$ WD model from Salaris et al. (1997) calculations (solid line), and after chemical redistribution upon crystallization (dashed line). Right : Time delay due to the chemical redistribution, from the models by Salaris et al. (2000).

with time. Given that most stars are or will become WDs, plus the existence of a well defined relationship between cooling time and luminosity, and the long cooling timescales, WDs are very attractive candidates to unveil the history of star formation in the Galaxy. During the last two decades observations and theory have improved to a level that has made finally possible to employ WDs for determining ages of the stellar populations in the solar neighborhood, and in the nearest star clusters.

\section{White Dwarf evolution}

The main phases of WD cooling evolution are briefly sketched below. The figures for the luminosity ranges are just indicative, for more precise values depend on the exact chemical stratification in the core and chemical composition of the non-degenerate envelope.

$\log \left(L / L_{\odot}\right)>-1.5$. The brightest stages of WD evolution are dominated by neutrino emission (mainly plasma-emission). By the end of this phase of neutrino cooling, differences in the thermal structures at fixed mass, due to different approaches to the WD cooling sequence disappear. If the mass in the H-envelope is above a threshold of $\approx 10^{-4} M_{W D}$ (the exact value depending on the WD mass), hydrogen burning through the $p p$ chain becomes effective. Pulsational studies seem to constrain the $M_{H}$ to values generally below this threshold, see, e.g., Castanheira \& Kepler (2008).

$-3<\log \left(L / L_{\odot}\right)<-1.5$. The main source of energy is the internal energy of the ions. The Coulomb parameter $\Gamma$ in the core is above unity, i.e. the ions are in the liquid phase.

$\log \left(L / L_{\odot}\right)<-3.0$. The Coulomb parameter $\Gamma$ reaches the critical value $\Gamma_{\text {cryst }} \sim 180$, and the ions in the core undergo a phase transition from liquid to solid. This introduces two new energy sources. The first one is the latent heat of crystallization, of the order $K_{B} T$ per crystallized ion, where $K_{B}$ is the Boltzmann constant. The second source is related to the phase diagram of the $\mathrm{CO}$ binary mixture, see, e.g., Isern et al. (2000). In brief, the equilibrium composition of the $\mathrm{CO}$ mixture of the solid and liquid phases are not the same. The net effect is a migration of oxygen towards the central regions with the consequent release of gravitational energy (see, Fig. 1). 

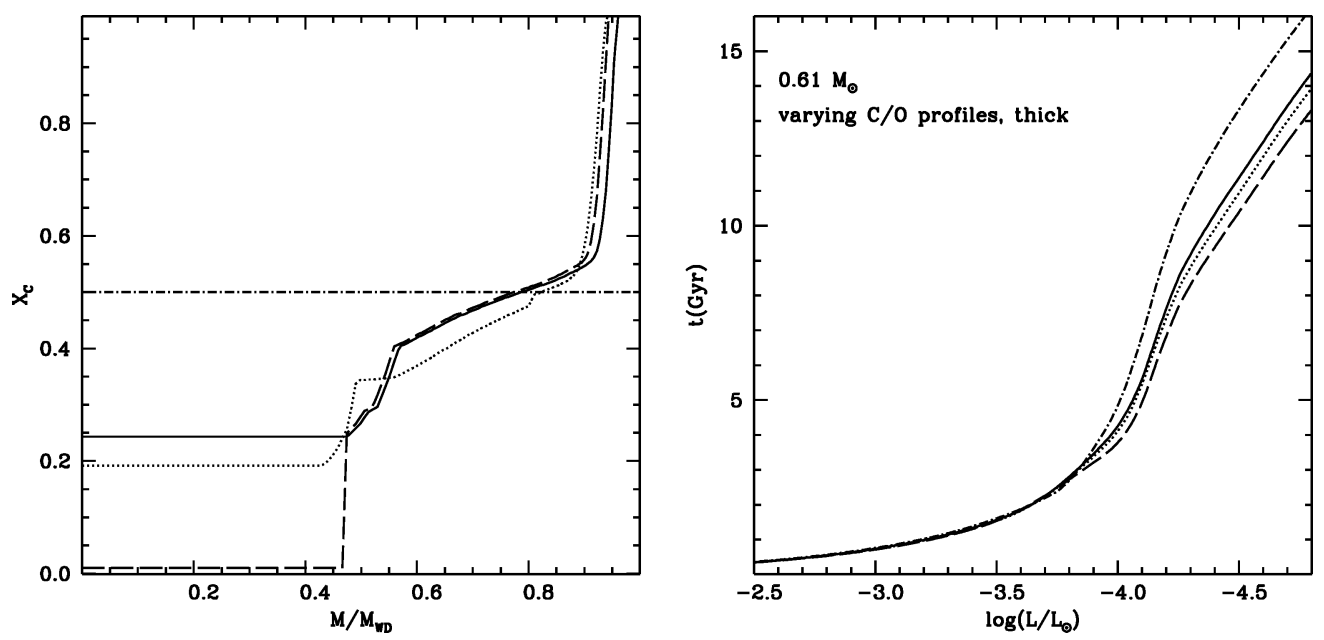

Figure 2. Left: Test carbon profiles in the core of a $0.61 M_{\odot}$ model. Right : Corresponding cooling timescales (see text for details).

Debye cooling. When crystallization of the CO core is essentially complete, the specific heat follows the Debye law, decreasing as $T^{3}$. At this stage, the energy provided by the compression of the non-degenerate envelope (through the virial theorem) is enough to prevent the sudden disappearance of WDs with sufficiently thick envelopes.

The local detailed energy budget of a WD is given by, as shown in Isern et al. (1997):

$$
-\left(\frac{d L_{\mathrm{r}}}{d m}+\epsilon_{\nu}\right)=C_{\mathrm{v}} \frac{d T}{d t}+T\left(\frac{\partial P}{\partial T}\right)_{V, X_{0}} \frac{d V}{d t}-l_{\mathrm{s}} \frac{d M_{\mathrm{s}}}{d t} \delta\left(m-M_{\mathrm{s}}\right)+\left(\frac{\partial E}{\partial X_{0}}\right)_{T, V} \frac{d X_{0}}{d t}
$$

where $E$ is the internal energy per unit mass, $L$ the local luminosity, $\epsilon_{\nu}$ the neutrino energy losses per unit mass, $V=1 / \rho$ is the specific volume. The first term in the righthand side is the heat capacity of the star, the second one denotes the energy contribution due to changes in volume, which is usually negligible, apart from the latest stages of cooling. The third term represents the energy contribution due to the latent heat release upon crystallization ( $l_{\mathrm{s}}$ is the latent heat of crystallization and $d M_{s} / d t$ is the rate of growth of the solid core due to crystallization). The delta function specifies that the latent heat is released at the solidification front. The last term denotes the energy released by the change of chemical abundances (chemical separation) due to the phase diagram of the CO mixture. $X_{0}$ here represents the oxygen mass fraction. Figure 1 displays the effect of chemical separation upon crystallization on a $0.61 M_{\odot}$ model, with $M_{H}=10^{-4} M_{W D}$ and $M_{H e}=10^{-2} M_{W D}$. The delay in the cooling process caused by this phenomenon is comparable to the effect of latent heat release.

Apart from uncertainties in the input physics (equation of state, opacities, CO phase diagrams), the detailed energy budget is strongly dependent on the chemical stratification of the degenerate core and of the envelope. Varying the chemical composition of the core affects the internal energy available to be radiated away, the latent heat release and chemical redistribution upon crystallization. Varying the composition and mass thickness of the envelope affects the rate of energy release because of the change of opacity. In general, more transparent envelopes (e.g. thinner or absent H-layers) speed up the cooling process. To date, predictions from stellar evolution calculations are subject to sizable uncertainties regarding the $\mathrm{CO}$ profiles and envelope stratification at the onset of WD 

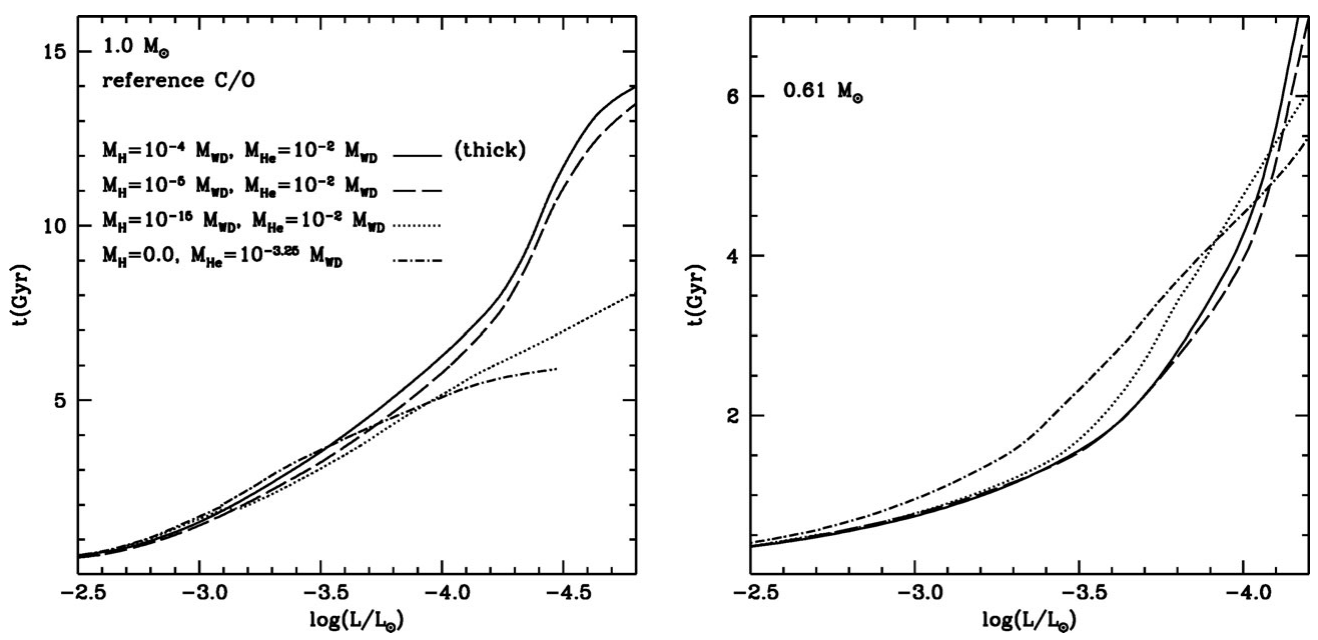

Figure 3. Left : Cooling timescales for a $1.0 M_{\odot}$ WD model with varying envelope properties. Right: As in the left panel but for a $0.61 M_{\odot}$ WD model.

cooling. Regarding the $\mathrm{CO}$ profile, the main reason is not only the uncertainty in the ${ }^{12} C+\alpha$ reaction rate - estimated to be of the order $1 \sigma= \pm 30 \%$ according to the latest determination by Kunz et al. (2002) - but also, and probably even more importantly, the treatment of core convection during the central He-burning phase. As discussed in detail by Straniero et al. (2003), for a given stellar mass, whether or not semiconvection is included, whether or not breathing pulses are suppressed and the way in which they are suppressed can alter substantially the final CO profile. There are good observational reasons why breathing pulses should be suppressed in model computations - e.g. Cassisi et al. (2003) - but the way in which they are suppressed can affect substantially the $\mathrm{CO}$ abundances. As for the envelopes, uncertainties in the mass-loss efficiency during the Asymptotic Giant Branch (AGB) phase do not allow a firm prediction of their chemical composition and thickness at the start of WD cooling. The uncertainty in the mass-loss along the AGB affects also the CO stratification in a more subtle way. Changing the massloss efficiency affects the initial (main sequence)-final (WD) mass relationship (IFMR), hence the relationship between the mass of the $\mathrm{CO}$ core (and associated chemical profile) at the end of central He-burning and its final value at the end of the AGB.

Figure 2 shows numerical tests that give an order-of-magnitude idea of uncertainties on cooling times due to alternative CO profiles. The cooling times of a $0.61 M_{\odot}$ WD with $M_{H}=10^{-4} M_{W D}$ and $M_{H e}=10^{-2} M_{W D}$ are calculated assuming 4 different chemical profiles in the core. The solid line represents the reference profile of Fig. 1, the dasheddotted line shows a flat $\mathrm{CO}$ profile with equal abundances (that maximizes the effect of chemical separation), the dashed line shows the effect of an alternative treatment of He-burning core convection following the results by Straniero et al. (2003), the dotted line accounts for a different IFMR (progenitor with a smaller initial mass). The largest effect compared to the reference model is obtained with the flat $\mathrm{CO}$ profile, that causes an age increase up to 1.5-2 Gyr for the oldest WDs. The other profiles cause generally a reduction of cooling times by at most $\sim 1$ Gyr.

Figure 3 displays the cooling times of a $1.0 M_{\odot}$ with the CO stratification by Salaris et al. (1997) and different envelopes. The model without H-layer is representative of a non-DA WD, and has the fastest cooling time, because of a lower envelope opacity. 
Age differences up to $\sim 8$ Gyr appear for the faintest observed WDs $\left(\log \left(L / L_{\odot}\right) \sim-4.5\right)$. The case with $M_{H}=10^{-15} M_{W D}$ deserves a further discussion. In this model, surface convection (efficient below a certain effective temperature) has been inhibited, so that the outermost H-layer is never mixed with the underlying, much thicker He-layer. If mixing is allowed, the H-layer is engulfed by the He envelope at temperatures well above $10000 \mathrm{~K}$, when the cooling timescales of DA and non-DA models are essentially the same. From that moment on the model behaves as the non-DA calculations shown in the figure, because the hydrogen is completely diluted within the He-envelope - see also the discussion in D'Antona \& Mazzitelli (1987). Thicker H-layers escape mixing with the underlaying He-envelope (for example $M_{H}=10^{-4}-10^{-5} M_{W D}$ ), or mix at lower luminosities. The right-hand panel of the same figure shows the case of a $0.61 M_{\odot}$. Notice how for cooling ages between $\sim 1$ and $\sim 4$ Gyr the model with pure He envelope is sizably brighter than models with an external H-layer, whereas this effect is smaller for the $1 M_{\odot}$ model. A surface H-layer with $M_{H} \approx 10^{-10} M_{\odot}$ would mix at $T_{\text {eff }} \sim 9000 \mathrm{~K}$, i.e. $\log \left(L / L_{\odot}\right) \sim-3.0$, and after the mixing episode the cooling timescales will run parallel to the case of the non-DA model.

\section{White dwarf cosmochronology}

Fundamental working tools for WD age dating are WD isochrones, i.e. the CMD of WDs born from a single-burst, single-metallicity population (see, Fig. 4). They are routinely used to study the cooling sequences of WDs in star clusters, see, e.g., Hansen et al. (2007), Bedin et al. (2008a). Computations of WD isochrones require a grid of WD models for different masses, an IFMR and evolutionary timescales of the WD progenitors plus appropriate bolometric corrections. From a WD isochrone, the luminosity function (LF) in a given passband can be calculated after assuming an initial mass function (IMF) for the WD progenitors. Here the reference WD isochrones are computed using the cooling models by Salaris et al. (2000) that make use of Salaris et al. (1997) CO profiles, envelopes with $M_{H}=10^{-4} M_{W D}$ and $M_{H e}=10^{-2} M_{W D}$, and progenitor lifetimes for a metal mass fraction $\mathrm{Z}=0.0198$ (solar) from Pietrinferni et al. (2004). The adopted IFMR is displayed in Fig. 5 as a solid line. The reference LFs are computed using a Salpeter IMF for the WD progenitors. Figure 4 displays, as an example, four isochrones and the corresponding LFs in the F606W ACS filter. The age indicator for a WD population is the faint end of the isochrones, that corresponds to a cut-off in the luminosity function. Due to the finite age of the stellar population, the more massive WDs formed from higher-mass and shorter-lived progenitors pile up at the bottom of the cooling sequence, producing the turn to the blue (i.e. a turn towards lower radii) visible at the faint end of the isochrones. Increasing the age obviously makes the bottom end of the isochrones fainter, because of the longer cooling times. The F606W magnitude of the LF cut-off changes with age by $\sim 0.2 \mathrm{mag} / \mathrm{Gyr}$ at old ages, and $\sim 0.5 \mathrm{mag} / \mathrm{Gyr}$ at intermediate ages. This in principle makes WDs better suited for age determinations compared to the main sequence turn off (the corresponding changes of $\mathrm{F} 606 \mathrm{~W}$ for the turn off are $\sim 0.1 \mathrm{mag} / \mathrm{Gyr}$ and $\sim 0.4 \mathrm{mag} / \mathrm{Gyr}$, respectively), once the cluster distance modulus is fixed.

Figure 5 shows the sensitivity of the LF to changes in the adopted IFMR. The two IFMRs used in the test are well within the scatter of points that defines semiempirical estimates for objects belonging to the Galactic Disk system (field stars and open clusters). The full IFMR (especially the high-mass end) of old star clusters is, from an empirical point of view, largely unknown. The shape of the resulting LFs is severely affected 

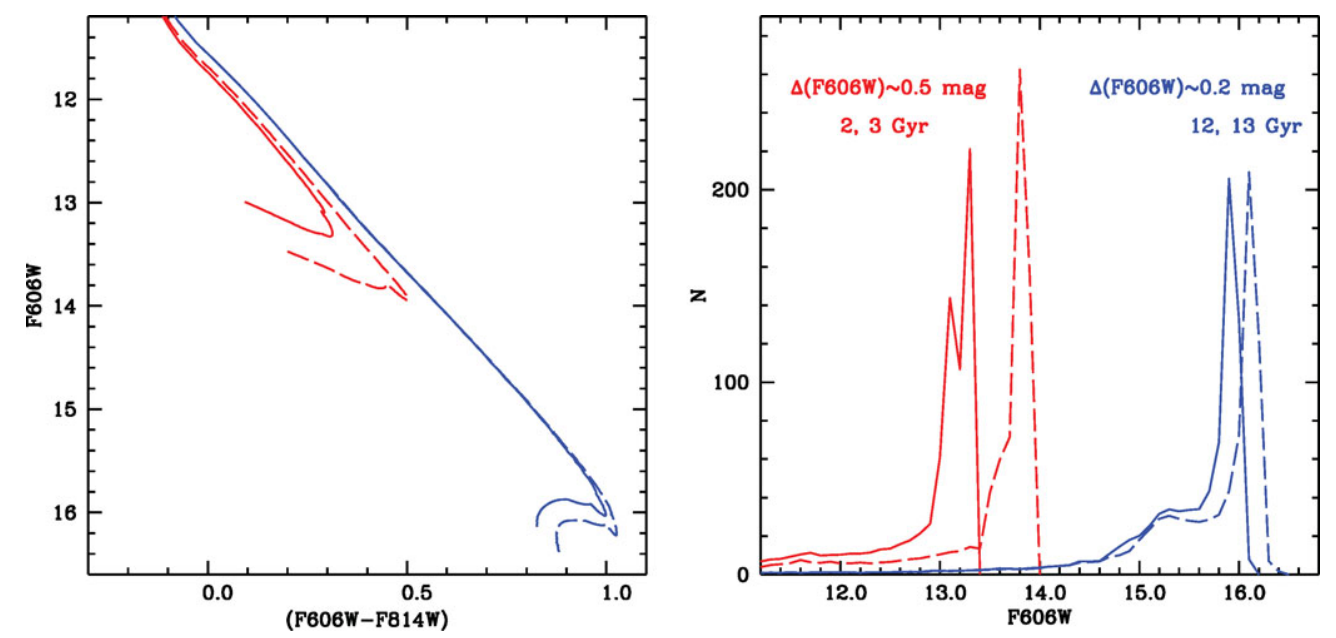

Figure 4. Left: WD isochrones for 2, 3, 12, 13 Gyr. Right : Luminosity functions in the F606W ACS filter, for the isochrones in the left panel.

by the choice of IFMR. Bumps and plateaus do appear, depending on the age of the population. The magnitude of the cut-off is only moderately affected for the older ages. If one compares just the observed magnitude of the LF cut-off with theory, these two different IFMRs cause an age uncertainty below $10 \%$ for the two oldest ages displayed. The effect for the youngest LF is larger. Taking into account the overall shape of the LF would put firm constraint on the form of the IFMR and this should mitigate this source of indetermination for the WD ages. The matter is however complicated by the fact that a change of the progenitor IMF also alters the overall shape of the LF, as well as photometric errors and the presence of unresolved WD+WD binaries, this latter point being discussed in some detail by Bedin et al. (2008b). Additionally, the transition of a fraction of DA objects to non-DA could also cause some local change in the shape of the $\mathrm{LF}$, because of the change of evolutionary speed if the mixing happens at $T_{\text {eff }}$ below $\approx 10000 \mathrm{~K}$.

The assumed CO stratification and envelope chemical composition are more troublesome from the point of view of age estimates, as shown in Fig. 6, that displays the effect of changing the $\mathrm{CO}$ profile from the reference case to a flat one. At old ages, LFs with 1.5 Gyr age differences are close to indistinguishable, provided that the progenitor IMF is a power law with exponent $\alpha=-1.7$, instead of $\alpha=-2.35$ (Salpeter). Given that the progenitor IMF - or, more precisely, the progenitor mass function MF that could be different from the IMF because of the dynamical evolution of the stellar system is at some level an unknown quantity in the observed cluster fields, this degeneracy between theoretical LFs with different ages is worrying. An analogous result is found when comparing the reference case with the LF computed from models with a thinner H-layer $\left(M_{H}=10^{-5} M_{W D}\right)$. Figure 6 shows how at old ages a systematic error of at least 0.8 Gyr seems to be unavoidable given our inability to predict the envelope thickness and composition of WDs.

One could try - if allowed by the quality of the photometric data at hand, see e.g. Hansen et al. (2007) - to use the full CMD to break these age degeneracies. Figure 7 compares reference isochrones with the case of a flat $\mathrm{CO}$ profile and a thinner H-layer, respectively. Notice how the degeneracy of the LF is mirrored by a near degeneracy in the CMD. To assess whether it is possible to use the distribution of points in the 

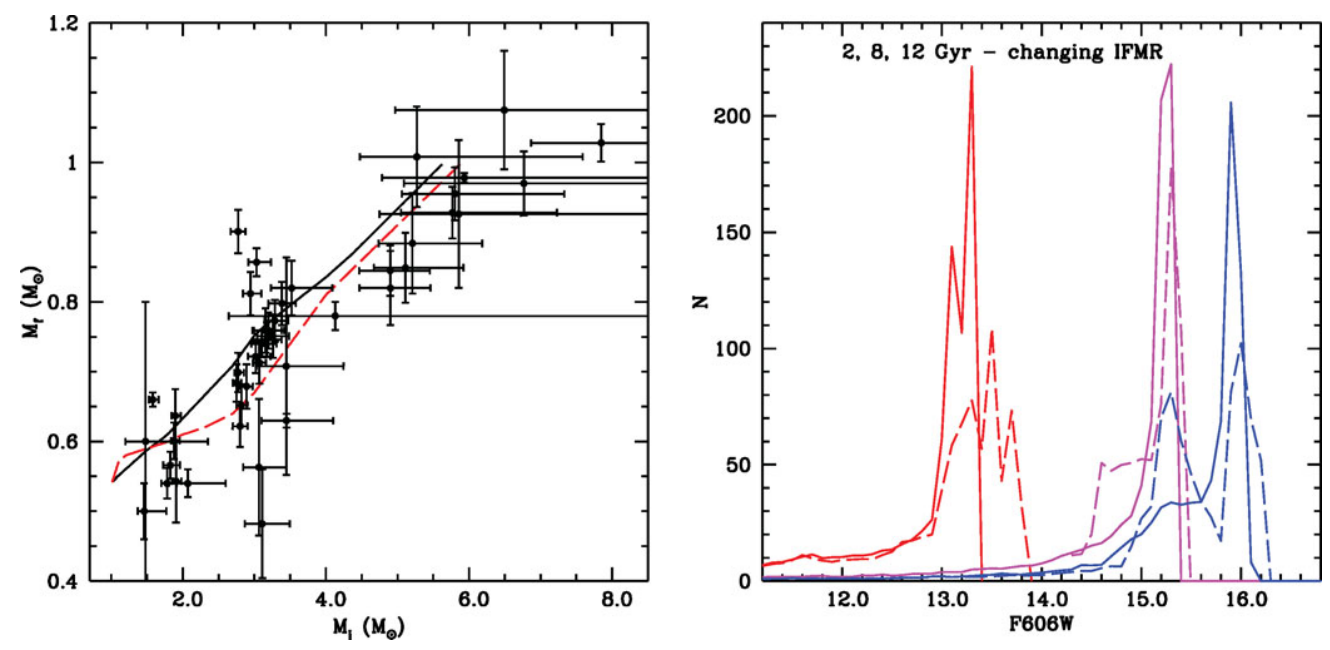

Figure 5. Left: IFMRs adopted in the calculations presented in this paper. The solid line is the reference IFMR. Datapoints with error bars are semiempirical estimates from Salaris et al. (2009) and, at low progenitors masses, from Catalan et al. (2008a), for WDs in open clusters and in the field. Right : Effect of varying the IFMR on the LF of star clusters with the labelled ages. The different line-styles correspond to the two IFMRs in the left panel.
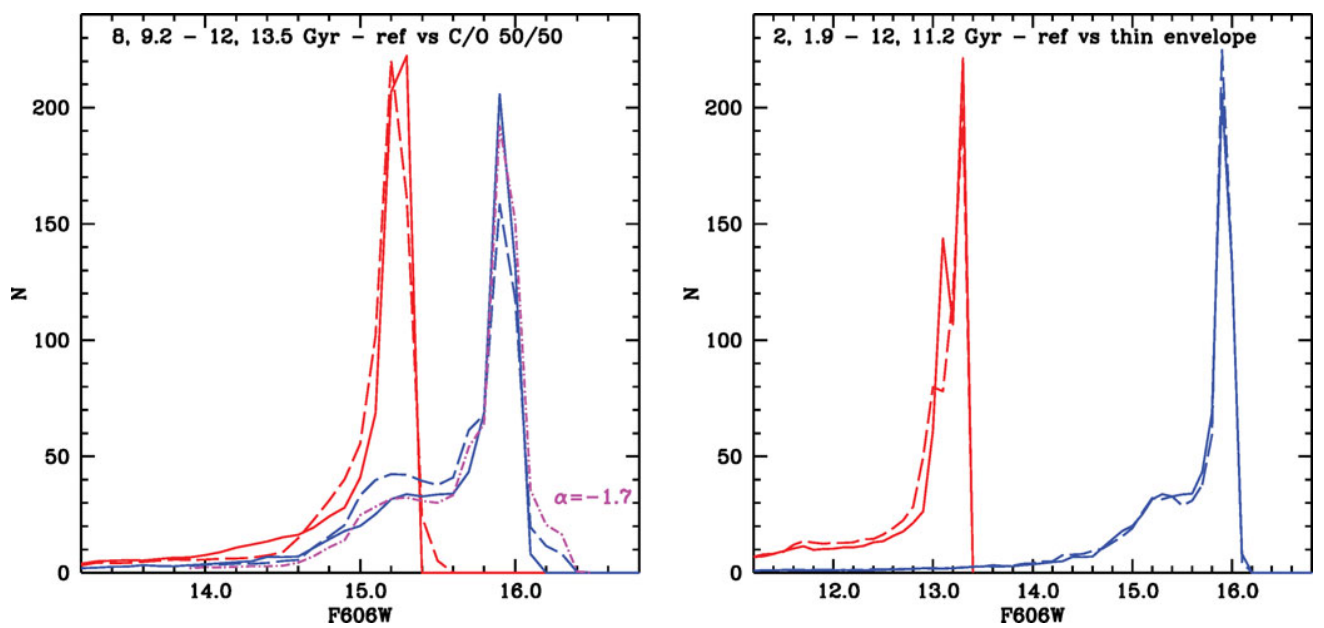

Figure 6. Left: Theoretical LFs with the labelled pairs of ages, for the reference case (solid lines) and for models with a flat CO profile (dashed lines). The case of a flat CO profile and a progenitor mass function with exponent $\alpha=-1.7$ is displayed as a dashed-dotted line. Right : As for the left panel, but for the case of reference (solid line) versus a thinner $\left(M_{H}=10^{-5} M_{W D}\right)$ H-layer (dashed line).

CMD to break the age degeneracy, the following test has been performed. Photometric errors and completeness as a function of F606W from the (F606W, F814W) photometry of NGC 6791 by Bedin et al. (2008a) have been considered. The reference F606W magnitudes have been then shifted so that the $50 \%$ completeness level is reached at $\mathrm{F} 606 \mathrm{~W}=16$, i.e. a luminosity on the descending branch of the LF cut-off in Fig. 6. This is a reasonable assumption for the existing photometries of old clusters. Monte-Carlo simulations have been then employed to produce synthetic samples of WDs from the pairs of old isochrones in Fig. 7, that include the prescribed photometric error law. A 

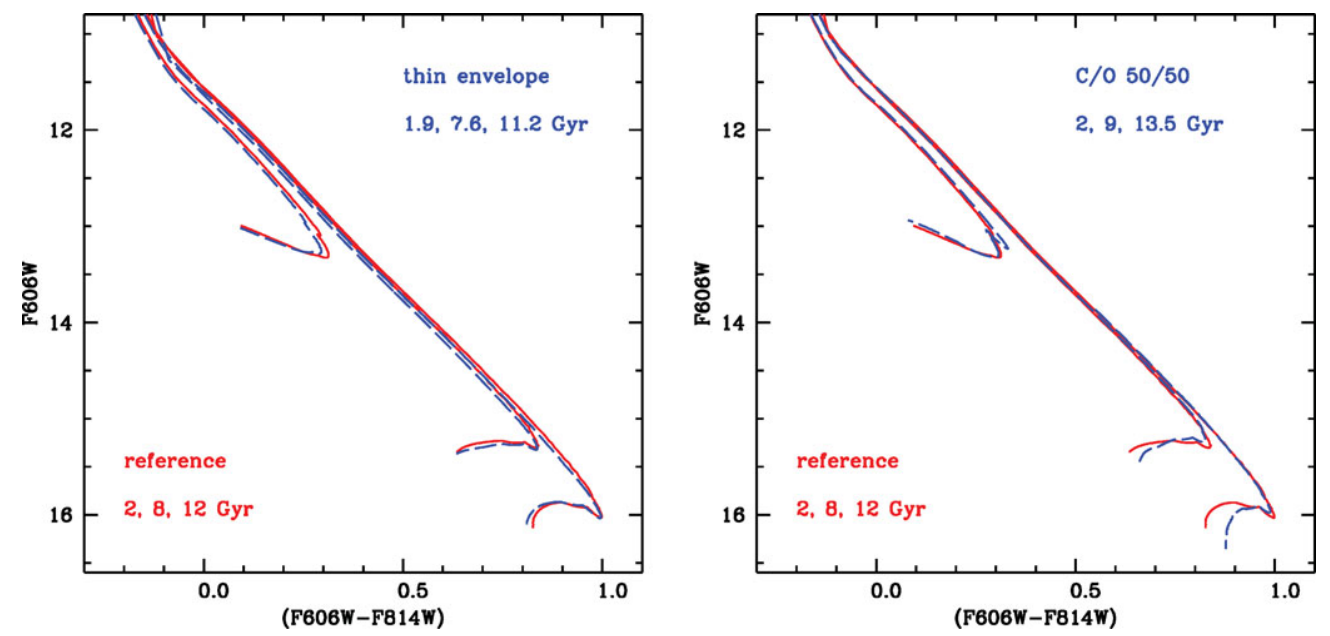

Figure 7. Left : Isochrone for the reference case and the labelled ages (solid lines) versus isochrones with a thinner $\left(M_{H}=10^{-5} M_{W} D\right)$ H-layer (dashed lines) and the three labelled ages. Right: As in the left panel but for the case of reference (solid lines) versus flat CO profiles (dashed lines).

2-dimensional KS-test applied to several realizations of the two pairs of synthetic WDs (sample size equal to 400 objects, but the precise value is not critical) for $\mathrm{F} 606 \mathrm{~W} \leqslant 16$ gives probabilities well below 95\% (assumed as threshold probability) that the WD populations in each pair are actually generated by two different distributions. Halving the errors at a given magnitude would produce again the same result for the case of thinner H-layers. A fainter (by about 0.1-0.2 mag) magnitude limit would allow a discrimination between the reference and flat $\mathrm{CO}$ case.

The presence of a fraction of non-DA objects (e.g. up to 30-40\%) modelled with pure-He envelopes does not affect sensibly the LF of old populations, because at those ages nonDA objects would be mainly located at much fainter magnitudes, well below detectability. However, their effect on the LF may be significant for ages of 2-3 Gyr, that correspond to luminosities where the cooling timescales of WDs with pure He-envelopes are longer than the case of H-envelopes. The situation may become more complicated in case a sizable fraction of DA objects have a range of H-layer masses, and undergo mixing with the He-layer at luminosities where the evolutionary speed is very different for $\mathrm{H}$ - and He-envelopes. In this case, additional features in the LF (and stellar distribution on the CMD) can appear, related to the change in evolutionary timescales.

Regarding the age of field WDs, investigations are traditionally focused on the LF of WDs in the solar neighborhood, see, e.g., Leggett et al. (1998). Figure 8 displays an observational LF from Catalan et al. (2008b), compared to the theoretical counterpart calculated from the reference models described above, solar metallicity progenitors, a Salpeter IMF and a continuous star formation rate. All theoretical LFs are normalized to the observed star counts at $\log \left(L / L_{\odot}\right)=-3.2$. The position of the cut-off in the empirical LF provides an age $t=11.5 \pm 1.0 \mathrm{Gyr}$ for the onset of star formation in the local Disk. The right panel of Figure 8 shows also the result for models with a flat CO profile and a thinner H-layer $\left(M_{H}=10^{-5} M_{W D}\right)$ respectively. The systematic change in age is essentially the same as for the case of the star counts from isochrones discussed above. The left panel displays also the case of a substantial primordial fraction of DB objects, taken as representative of the whole non-DA population. The effect is at the level 

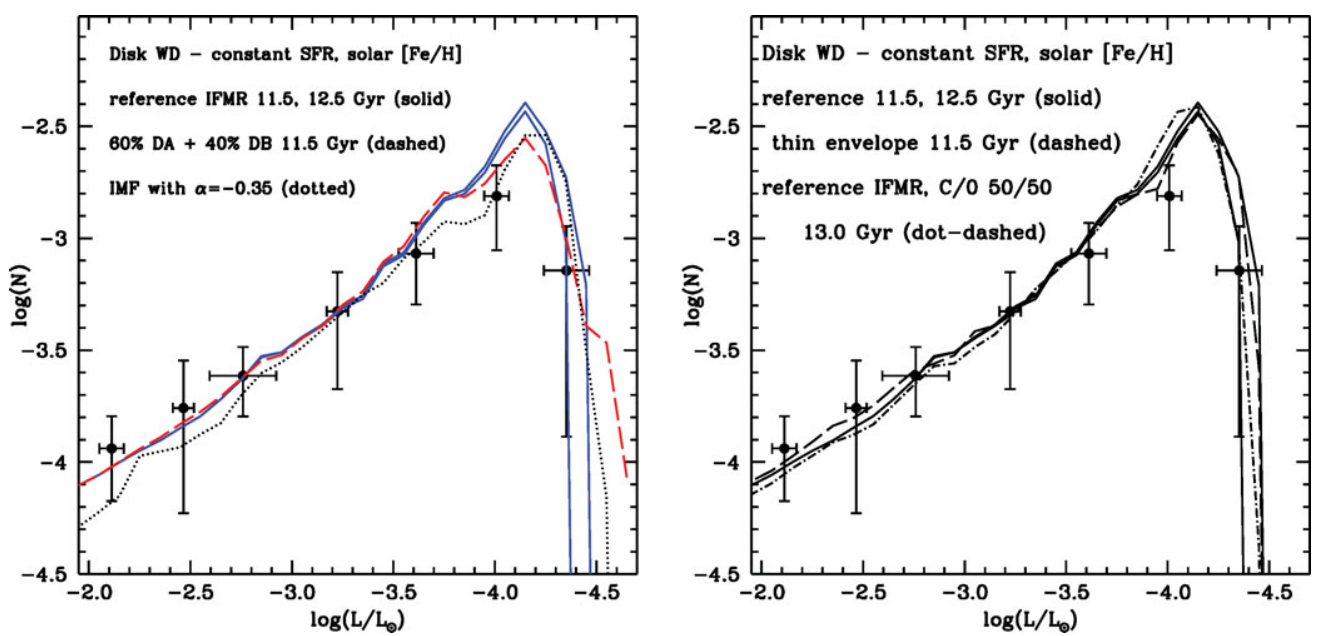

Figure 8. Left: Observed LF for WDs in the solar neighborhood from Catalan et al. (2008b) compared, respectively, to the reference DA theoretical counterparts for the labelled ages, a LF computed allowing for a $40 \%$ fraction of DB objects, and a LF with DA models but an IMF with exponent $\alpha=-0.35$. Right: As in the left panel, but with additional LFs obtained from models with a flat $\mathrm{CO}$ profile and with a thinner H-layer $\left(M_{H}=10^{-5} M_{W D}\right)$ respectively (see text for details).

of only a few $10^{8} \mathrm{yr}$, because of a different shape of the LF at the lower end. The faster cooling times of He-envelope models tend to distribute the fainter DB objects below the observed magnitude of the cut-off. Tremblay \& Bergeron (2008) data show that the transformation of DA into non-DA objects due to surface convection - that changes the observed non-DA/DA observed ratio from $\sim 0.25$ to $\sim 0.4-0.5-$ happens in local Disk objects at $T_{e f f} \sim 10000 \mathrm{~K}$, i.e. at a temperature where evolutionary timescales of DA and DB models are virtually the same. This means that an overall $40 \%$ primordial fraction of DB models is an adequate assumption, as far as the effect on the Disk LF is concerned. Changing the exponent of the IMF has also an effect on the age because of a different shape of the LF. The change applied to the theoretical LF in Fig. 8 causes an age change by $\approx 0.5$ Gyr. Finally, the effect of the alternative IFMR displayed in Fig. 5 causes a decrease of the derived age by less than 1 Gyr.

\section{Conclusions}

As discussed in some details in the previous sections, uncertainties in the CO stratification and envelope composition give probably the largest contribution to the systematic errors in age estimates from the WD LF. The analysis of the WD distribution across the CMD may not always help in removing these sources of uncertainties. Also the choice of the IFMR may play a non-negligible role in the final error budget on WD ages. Together with these systematics arising from the modelling of core convection and mass-loss during the previous evolutionary phases, uncertainties in the adopted input physics introduce additional sizable systematic differences in the cooling times calculated by different authors. Comparisons of a $0.6 M_{\odot}$ pure-C WD model by Fontaine et al. (2001) with results from the Salaris et al. (2000) code, using a simple gray $T(\tau)$ relationship for the surface boundary conditions, $M_{H}=10^{-4} M_{W D}$ and $M_{H e}=10^{-2} M_{W D}$, reveal differences of $\sim 1$ Gyr for cooling times of the order of 12-13 Gyr. Additional physics not yet included 
in age estimates of WD populations may be potentially responsible for other systematic effects. Deloye \& Bildsten (2002) and García-Berro et al. (2008) have explored the role played by ${ }^{22} \mathrm{Ne}$ diffusion in the liquid phase, concluding that for progenitor metallicities solar or supersolar and old ages $(\approx 10 \mathrm{Gyr})$, a $\approx 1$ Gyr age increase in estimates from the LF has to be accounted for. There are however still sizable uncertainties in the determination of the appropriate Ne diffusion coefficient. The effect of minor chemical species on the phase diagram of the WD core and on the time delays due to chemical separation is still not well established. Calculations by Segretain (1996) for a CONe ternary mixture and solar progenitor metallicity show a negligible effect compared to results for the CO binary mixture. The effect of the chemical separation of Fe has not been yet assessed from the appropriate multibody phase diagram, although, as summarized in Isern et al. (2002), estimates from an effective $\mathrm{N} / \mathrm{Fe}$ binary mixture (whereby $\mathrm{N}$ has the average charge of a flat CO profile) give a delay of the order of 1 Gyr for a $0.6 M_{\odot}$ WD, that is probably an upper limit to the true effect. Taken all together, these confirmed and potential sources of uncertainties show that WD evolution is not just a simple cooling problem that naturally provides ages more accurate than, e.g. main sequence turn off ages. Instead, it is testing the models and - as long as ab-initio solid theoretical predictions are still lacking - tuning the uncertain inputs against turn off ages in star clusters, that should be the most fruitful approach to firmly establish WD cosmochronology as a useful tool on its own.

\section{References}

Bedin, L. R., King, I. R., Anderson, J., Piotto, G., Salaris, M., Cassisi, S., \& Serenelli, A. 2008, ApJ, 678, 1279

Bedin, L. R., Salaris, M., Piotto, G., Cassisi, S., Milone, A. P., Anderson, J., \& King, I. R. 2008, ApJ, 679, L29

Castanheira, B. G. \& Kepler, S. O. 2008, MNRAS, 385, 430

Cassisi, S., Salaris, M., \& Irwin, A. W. 2003, ApJ, 588, 862

Catalán, S., Isern, J., García-Berro, E., Ribas, I., Allende Prieto, C., \& Bonanos, A. Z. 2008a, $A \mathscr{E} A, 477,213$

Catalán, S., Isern, J., García-Berro, E., \& Ribas, I. 2008b, MNRAS, 387, 1693

D'Antona, F. \& Mazzitelli, I. 1987, in The Second Conference on Faint Blue Stars, D.S. Hayes and J.W. Liebert (eds.), L. Davis Press, p. 635

Deloye, C. J. \& Bildsten, L. 2002, ApJ, 580, 1077

Fontaine, G., Brassard, P., \& Bergeron, P. 2001, PASP, 113, 409

García-Berro, E., Althaus, L. G., Córsico, A. H., \& Isern, J. 2008, A\& A, 677, 473

Hansen, B. M. S. \& Liebert, J. 2003, ARAE A, 41, 465

Hansen, B. M. S., et al. 2007, ApJ, 671, 380

Kunz, R., et al. 2002, ApJ, 567, 643

Isern, J., Mochkovitch, R., García-Berro, E., \& Hernanz, M. 1997, ApJ, 485, 308

Isern, J., García-Berro, E., Hernanz, M., \& Chabrier, G. 2000, ApJ, 528, 397

Isern, J., García-Berro, E. , Hernanz, M., \& Salaris, M 2002, Contributions to Science, 2, 237

Leggett S. K., Ruiz M. T., \& Bergeron P. 1998, ApJ, 497, 294

Pietrinferni, A., Cassisi, S., Salaris, M., \& Castelli, F. 2004, ApJ, 612, 168

Salaris, M., Dominguez, I., García-Berro, E., Hernanz, M., Isern, J., \& Mochkovitch, R. 1997, ApJ, 486, 413

Salaris, M., García-Berro, E., Hernanz, M., Isern, J., \& Saumon, D. ApJ, 544, 1036

Salaris, M., Serenelli, A., Weiss, A., \& Miller Bertolami, M. 2009 ApJ, in press

Segretain, L. 1996, A\& $A$, 310, 485

Straniero, O., Dominguez, I., Imbriani, G., \& Piersanti, L. 2003, ApJ, 583, 878

Tremblay, P.-E. \& Bergeron, P 2008, ApJ, 672, 1144 


\section{Discussion}

G. Piotro: You have listed six sources of uncertainty in the age we can derive from the WD cooling sequence. How do they size up? Can we leave this room with an idea of the uncertainty in the cluster age derived from white dwarf cooling sequences?

M. SAlaris: It is difficult to say with some precision. $10^{-4} M_{\mathrm{WD}}$ is an upper limit to the H-layer thickness. So, an uncertainty there goes always in the direction of reducing cooling timescales. The effect of $\mathrm{CO}$ profiles can go both ways. ${ }^{22} \mathrm{Ne}$ diffusion goes in the direction of always increasing $t_{\text {cool }}$. Uncertainties due to different input physics may probably go both ways. I would say that globally we have something of the order of $+2-3$ to -2 Gyr at old ages. This is of course just a very rough estimate.

J. Melbourne: So you point out all the variations in the models, but what about the large potential variations in the stars themselves. Variations in He envelopes and CO profiles? How do you account for these uncertainties in your methods?

M. SALARIS: If the mixing treatment, the initial-final mass relationship and the metallicities of the progenitors are specified, the CO profiles of WDs with different masses are uniquely defined. The problem is the envelope; that depends on the details of the mass loss at the level of $10^{-2}$ to $10^{-4} M_{\odot}$. One has to make assumptions, treating the $\mathrm{H}$ and He layer thickness essentially as free parameters (of course within certain limits).

S. LEGGETT: What is the importance of star formation history or the age of the disk as implied by the luminosity function?

M. SAlARIS: This is a very good point. The somewhat standard assumption is a constant SFR with solar metallicity progenitors. I suspect the effect is less important than uncertainties in the CO profiles and envelope thickness. Matt Wood (1992, ApJ, 386, 539) has investigated this issue. From his Figures 13 and 15 it transpires that the age of the disk derived from the luminosity function cut-off is not greatly affected by the choice of the SFR, at least for the cases analyzed in his paper.

P. Demarque: What are the chances of learning about the core helium buring phase from WD observations? How does diffusion complicate things?

M. SALARIS: Comparisons between cooling ages and turn- off ages of clusters may give indications about the internal CO stratification, hence about He-burning core mixing. Also, studies of pulsating WDs can put constraints on the core $\mathrm{C} / \mathrm{O}$ ratios. Diffusion should not affect the $\mathrm{CO}$ profile but it is important to explain the lack of metals in the envelopes of WDs. Also, ${ }^{22} \mathrm{Ne}$ diffusion in the liquid phase alters the ${ }^{22} \mathrm{Ne}$ profile in the core. 


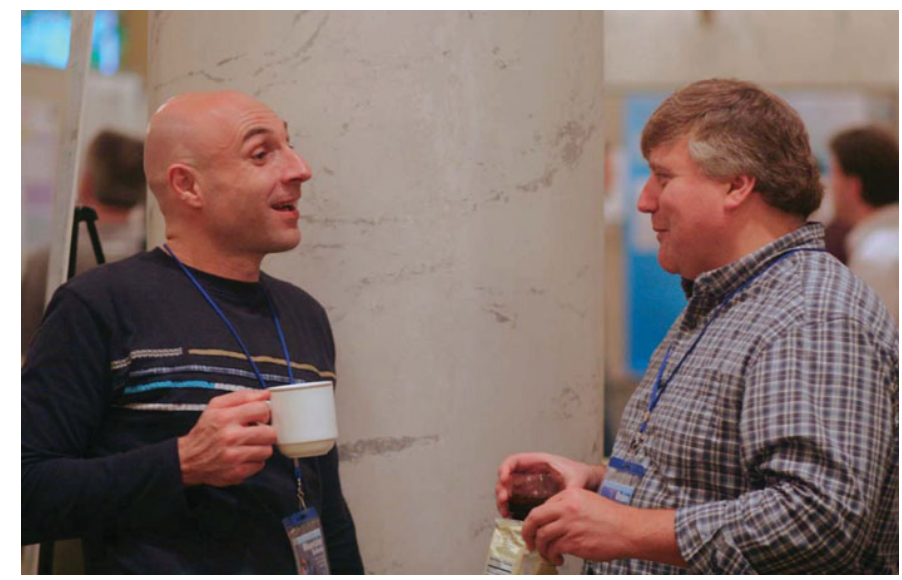

Maurizio Salaris and Brian Chaboyer

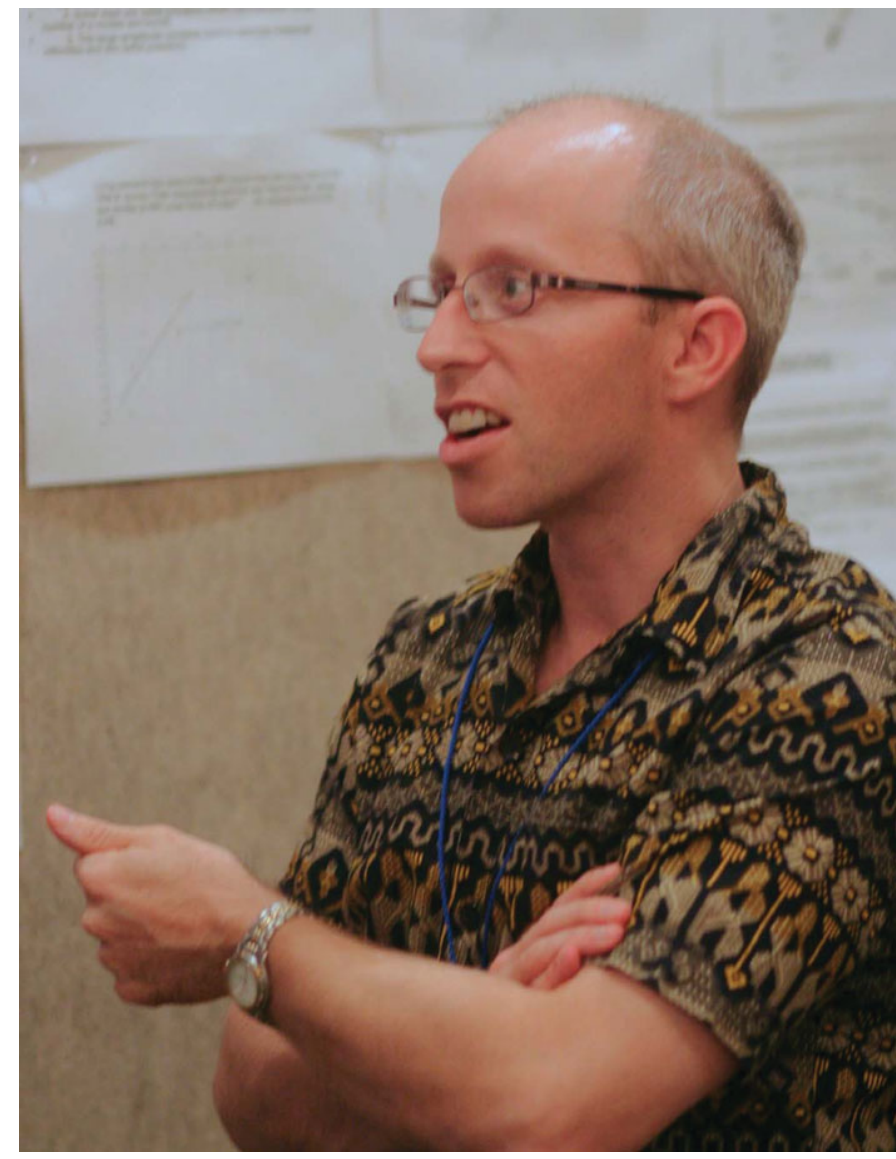

Jason Melbourne 